\title{
Fixação do enxerto do ligamento cruzado anterior no polo tibial: Análise biomecânica de três métodos*
}

\section{Fixation of the Anterior Ligament Graft at the Tibial Pole: Biomechanical Analysis of Three Methods}

\footnotetext{
${ }^{1}$ Faculdade Evangélica do Paraná (FEPAR), Curitiba, Paraná, Brasil

2 Programa de Pós-Graduação em Engenharia Mecânica e Materiais, Universidade Tecnológica Federal do Paraná (UTFPR), Curitiba, Paraná, Brasil

${ }^{3}$ Departamento de Ortopedia e Traumatologia, Hospital de Clínicas, Universidade Federal do Paraná (UFPR), Curitiba, Paraná, Brasil

${ }^{4}$ Departamento de Mecânica, Universidade Tecnológica Federal do Paraná (UTFPR), Curitiba, Paraná, Brasil

${ }^{5}$ Departamento de Ortopedia e Traumatologia, Universidade Federal do Paraná (UFPR), Curitiba, Paraná, Brasil
}

Fernando Pessoa Weiss ${ }^{1}$ Felipe Augusto de Aguiar Possoli ${ }^{2}$ Isabel Ziesemer Costa ${ }^{3}$ Paulo César Borges ${ }^{4}$ Edmar Stieven Filho ${ }^{50}$ Luiz Fernando Kubrusly ${ }^{1}$

\author{
Endereço para correspondência Edmar Stieven Filho, PhD, Rua \\ Francisco Rocha 62, sala 502, Batel, Curitiba, Paraná, Brasil, CEP: \\ 80.420-130 (e-mail: filho2000@gmail.com).
}

\section{Resumo}

\section{Palavras-chave}

- ligamento cruzado anterior

- dispositivos de fixação cirúrgica

- tíbia
Objetivo Análise biomecânica comparativa da resistência da fixação tibial para reconstrução ligamentar com parafuso de interferência, comparada com parafuso do tipo poste com arruela, e com fixação associada entre os métodos (fixação híbrida). Métodos Foram utilizados 54 corpos de prova (tíbia suína e tendão digital bovino), que foram divididos em 3 grupos com tipos de fixação semelhantes àqueles utilizados na reconstrução do ligamento cruzado anterior: 1) fixação com parafuso de interferência; 2) fixação com parafuso do tipo poste com arruela dentada sobre nó e fios de sutura; e 3) fixação com parafuso do tipo poste com arruela combinada com parafuso de interferência (fixação híbrida). Os testes foram realizados por meio de ensaios biomecânicos de tração tipo pull-out para determinação da rigidez e carga para falha (yield load) do sistema.

Resultados $O$ grupo com fixação híbrida apresentou maior rigidez final $(59,10 \pm 3,45 \mathrm{~N} / \mathrm{mm})$ do que os demais grupos $(p<0,05)$, e carga superior para falha $(581,34 \pm 33,48 \mathrm{~N})$ em relação ao grupo com parafuso de interferência $(p<0,05)$. Conclusão A fixação híbrida apresentou vantagens biomecânicas com relação ao sistema de fixação do enxerto de flexor digital bovino em tíbia suína durante os ensaios de tração.
Estudo realizado na Faculdade Evangélica do Paraná (FEPAR), e na Universidade Tecnológica Federal do Paraná (UTFPR), Curitiba, PR, Brasil. recebido

15 de Março de 2018 aceito

28 de Maio de 2018
DOI https://doi.org/ 10.1055/s-0039-1697015. ISSN 0102-3616.
Copyright $\odot 2019$ by Sociedade Brasileira License terms de Ortopedia e Traumatologia. Published by Thieme Revinter Publicações Ltda, Rio de Janeiro, Brazil 


\author{
Abstract \\ Keywords \\ - anterior cruciate \\ ligament \\ - surgical fixation \\ devices \\ - tibia
}

Objective Comparative biomechanical analysis of tibial fixation strength for ligament reconstruction with interference screw compared with screw post and washer, and compared with the associated fixation of both methods (hybrid fixation).

Methods A total of 54 specimens were used (porcine tibias and bovine flexor digital tendons), which were divided into three groups with fixation types similar to those used in anterior cruciate ligament ( $\mathrm{ACL}$ ) reconstruction: 1) fixation with interference screw; 2) fixation with screw post and toothed washer over knot and suture strand; and 3) fixation with screw post and washer combined with interference screw (hybrid fixation). The analyses were performed through pull-out biomechanical tensile tests to determine the stiffness and load to system failure (yield load).

Results The hybrid fixation group presented a significantly higher final stiffness $(59.10 \pm 3.45 \mathrm{~N} / \mathrm{mm})$ in comparison to the other groups $(p<0.05)$, as well as a higher yield load $(581.34 \pm 33.48 \mathrm{~N})$ compared to the interference screw group $(p<0.05)$. Conclusion Hybrid fixation had biomechanical advantages over the bovine digital flexor graft fixation system in swine tibia during tensile tests.

\section{Introdução}

O ligamento cruzado anterior (LCA) tem importância não apenas para a estabilidade ântero-posterior do joelho, mas também para toda a complexa cinética da marcha. ${ }^{1}$ Estudos americanos indicam uma incidência desta estrutura de 68,6/100.000 habitantes. ${ }^{2}$ A maior parte das lesões está relacionada com a prática de esportes, ${ }^{3}$ e, entre eles, o futebol é o que mais causa esse tipo de lesão. ${ }^{4}$

A resistência do LCA é muito próxima de $2.000 \mathrm{~N}$, mas mecanismos de torção do joelho em valgo e rotação interna são capazes de lesionar tanto o LCA quanto outras estruturas do joelho, como meniscos e cartilagem. ${ }^{5}$ Após a fase inflamatória inicial, os pacientes comumente experimentam uma sensação crônica de falseio associada à redução de sua capacidade funcional, o que em muitos casos exige a necessidade de intervenção cirúrgica. ${ }^{1}$

A técnica de reconstrução ligamentar permite o retorno dos pacientes às suas atividades esportivas. Os enxertos mais utilizados para reconstrução do LCA são osso-tendão-osso, retirado a partir do terço central do tendão patelar, e os tendões flexores do joelho (grácil e semitendíneo). ${ }^{6}$

Os métodos de fixação dos enxertos na reconstrução do LCA podem ser do tipo suspensão, poste, pressão, ou híbridos. Os métodos de poste promovem uma fixação cortical ao osso, mas podem usar como dispositivos intermediários suturas/fios, o que confere uma maior mobilidade do enxerto dentro do túnel. ${ }^{7}$ Já os métodos do tipo pressão promovem a fixação direta do tendão contra o osso esponjoso do túnel tibial, ou femoral, e mais próxima da articulação. ${ }^{8}$

A fixação tibial na reconstrução do LCA costuma ser um ponto de menor resistência do que a fixação femoral, em virtude da menor densidade óssea da tíbia, e pela fixação paralela do enxerto com o túnel. Isso gera uma força de escorregamento, podendo causar falha precoce da fixação distal., ${ }^{9} 10$

A integração do enxerto junto ao túnel ósseo ocorre por volta da décima segunda semana; ${ }^{11}$ por outro lado, a reabilitação fisioterápica precoce é importante no resultado clínico da cirurgia de reconstrução do LCA. Portanto, uma fixação segura no pós-operatório imediato é fundamental para prevenir deslocamentos adicionais e prejuízo ao processo de integração do enxerto. ${ }^{12}$

Considerando a possibilidade de falha relacionada aos métodos de fixação tibial da reconstrução do LCA, especialmente em relação ao parafuso de interferência isolado, ${ }^{13}$ métodos de fixação combinada, também chamados de fixação híbrida, passaram a ser difundidos. ${ }^{7}$ Desde então, vários estudos foram conduzidos a fim de determinar se o acréscimo de métodos efetivamente melhoraria a rigidez inicial do sistema. Neste aspecto, há divergência entre os estudos. ${ }^{10,14,15}$

Diante desta controvérsia, este trabalho foi conduzido por meio de ensaio de tração, em modelo animal, para comparar três dispositivos de fixação: o parafuso de interferência, o parafuso de tipo poste com arruela e amarrilho e a fixação híbrida (associação entre os dois métodos). A hipótese estudada foi a de que o método híbrido de fixação tem vantagens biomecânicas com relação aos métodos de pressão e poste isolados.

\section{Métodos}

Foram adquiridos em um frigorífico 54 tíbias suínas e 54 patas dianteiras bovinas, que foram cuidadosamente dissecadas a fim de obter o tendão digital profundo. Os tendões foram divididos ao meio, dispostos em pequenos recipientes plásticos contendo $50 \mathrm{~mL}$ de soro fisiológico (SF) a $0,9 \%$, e, então, armazenados e congelados a $-20^{\circ} \mathrm{C}$ até a data dos testes.

Para determinar a área da secção transversal dos tendões, estes foram posicionados em uma caixa de acrílico preenchida com pasta de Jeltrate tipo II de presa normal (Dentsply, York, PA, EUA). Os tendões foram envolvidos pela pasta até que esta atingisse consistência borrachuda, formando um molde. A pasta foi retirada dos modelos mantendo a impressão dos tendões. Esses moldes em alginato foram seccionados transversalmente em blocos com $10 \mathrm{~mm}$ de espessura, e digitalizados com resolução de 600 dpi por digitalizador HP J5780 (Hewlett- 
Packard, Palo Alto, CA, EUA). As áreas de secção transversal dos moldes foram medidas com auxílio do programa Image-Pro Plus (Media Cybernetics, Rockville, MD, EUA). ${ }^{16}$ Como os pares de tendões são dobrados ao meio para formar o enxerto quádruplo, as quatro menores áreas de cada um dos enxertos foram somadas para fins de cálculo formal de resistência, por ser a região de maior tensão sobre o enxerto. ${ }^{17}$

Os modelos foram mantidos em um ambiente com temperatura $\left(21^{\circ} \mathrm{C}\right)$ e umidade controladas por 24 horas antes dos procedimentos cirúrgicos, ${ }^{8}$ e mantidos hidratados até a conclusão dos ensaios de tração. Os tendões e tíbias foram divididos aleatoriamente entre os grupos. As extremidades dos tendões foram suturadas com a técnica de Krakow com 3 laçadas, ocupando $3 \mathrm{~cm}$ da extremidade de cada tendão, com fio cirúrgico de poliéster Ethibond Polyester 2 (Johnson \& Johnson, Piscataway, NJ, EUA).

Os três grupos foram então submetidos a procedimentos cirúrgicos: o primeiro grupo, com fixação com parafuso de interferência simples de $9 \times 30 \mathrm{~mm}(\mathrm{n}=19)$, chamado grupo interferência; o segundo, com fixação tipo poste com parafuso cortical ( $40 \mathrm{~mm}$ ) e arruela dentada sobre o nó da sutura $(n=18)$, chamado grupo poste; e o terceiro, com parafuso de interferência associado ao poste com parafuso e arruela dentada ( $\mathrm{n}=17$ ), chamado grupo híbrido (-Figuras 1 e 2 ).

Os túneis tibiais foram realizados com guia convencional a $55^{\circ}$, e perfurados com broca canulada de $9 \mathrm{~mm}$ de diâmetro. O enxerto foi passado pelo túnel até que $5 \mathrm{~cm}$ deste ficassem para fora da articulação, reproduzindo uma situação cirúrgica mais próxima da realidade.

Foram então montados no dispositivo de fixação composto por uma morsa de precisão angular com mordentes projetados para possibilitar a fixação de corpos cilíndricos, com três planos de liberdade, permitindo o alinhamento do túnel tibial com o eixo de tração da máquina. A porção óssea distal da tíbia foi fixada à maquina de tração por um tubo de policloreto de vinila (PVC) preenchido com epóxi (-Figura 3). ${ }^{18}$

Para os ensaios de tração, foi utilizada a máquina universal de ensaios EMIC DL 10000 (Instron, São José dos Pinhais, PR, Brasil), equipada com transdutores de deslocamento embutidos e uma célula de carga tipo "s" (CCE5KN, Instron, São José dos Pinhais, PR, Brasil ), com carga nominal máxima de 500 kgf e resolução de $0,1 \mathrm{kgf}$. Os resultados foram compilados por meio do programa Tesc (Instron, São José dos Pinhais, PR, Brasil), e extraídos de forma bruta (deslocamento

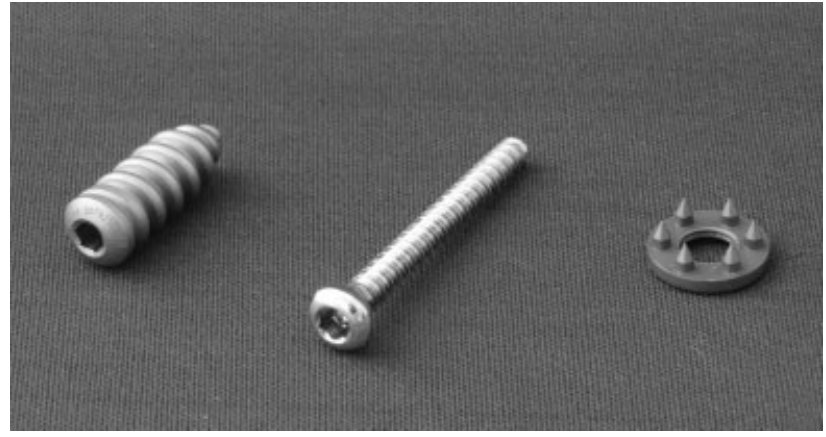

Fig. 2 Parafuso de interferência de $9 \times 30 \mathrm{~mm}$, parafuso cortical de $40 \mathrm{~mm}$, e arruela dentada.

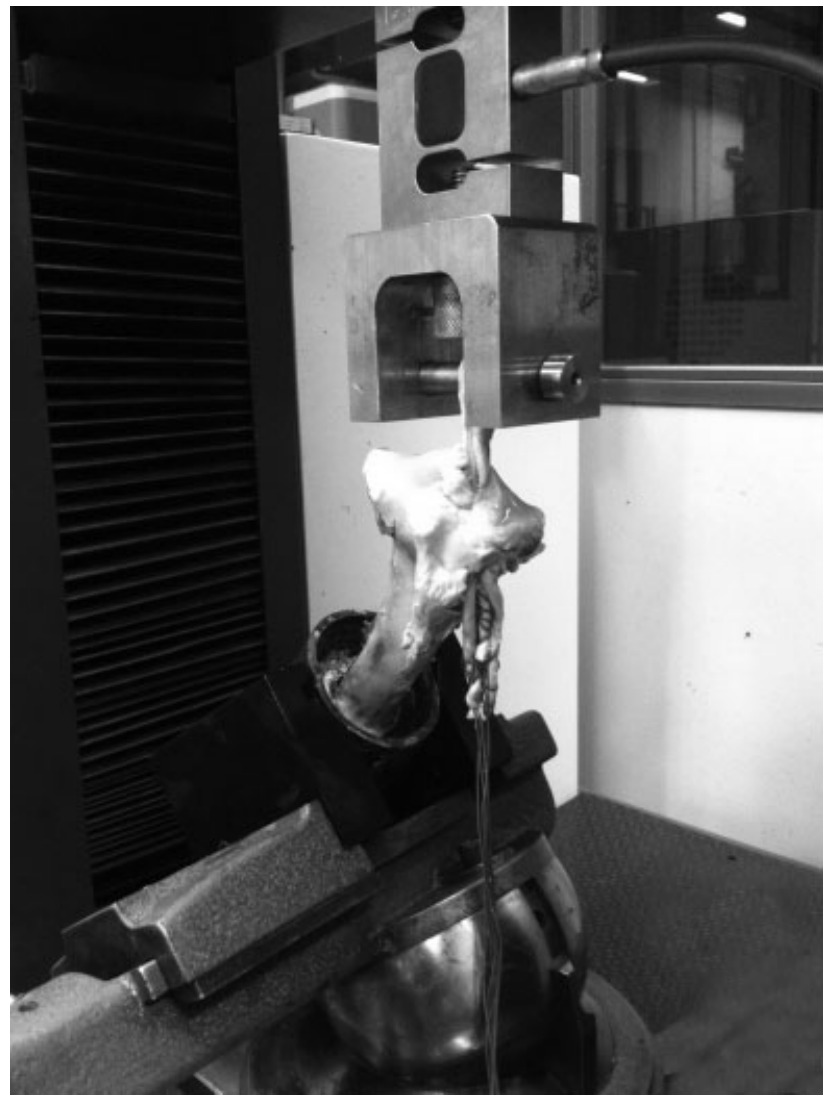

Fig. 3 Corpo de prova posicionado no dispositivo.
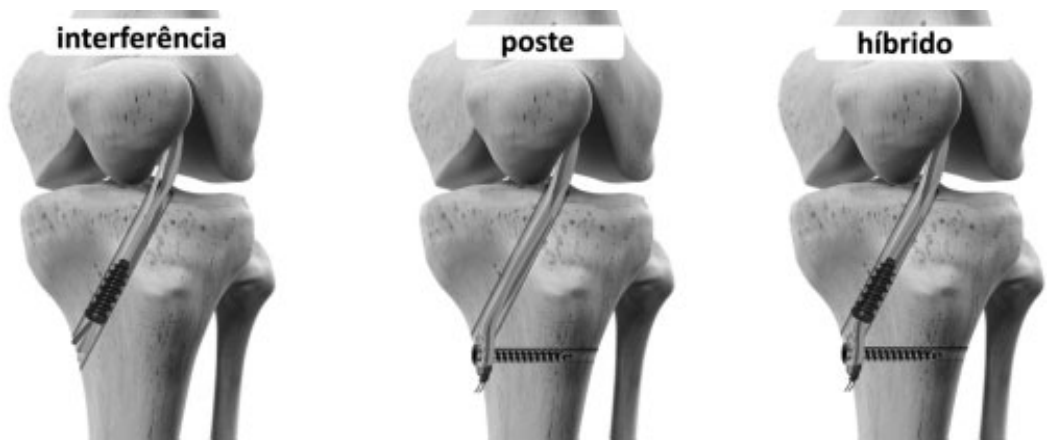

Fig. 1 Tipos de fixação (grupos). 
e força) para a análise gráfica e estatística em planilhas de Excel 365 (Microsoft Corp., Redmond, WA, EUA).

Realizou-se pré-tensionamento do enxerto com uma carga de $10 \mathrm{~N}$ durante 60 segundos, ${ }^{19}$ seguido pelo ensaio em velocidade de $50 \mathrm{~mm} / \mathrm{min}^{20}$ até a falha da fixação. Para análise dos resultados, foram utilizados a rigidez do sistema $\left(R_{R}\right)$ pelo método secante em baixas cargas (toe-region), a $R_{R}$ pelo método dos mínimos quadrados (tangente), e carga para falha do sistema (yield load), seguindo o coeficiente da correlação de Pearson ${ }^{10}$ e a tensão para falha $(\sigma R)$. 0 primeiro ponto da curva no qual o gráfico perde sua linearidade foi considerado a carga para falha (yield load), de forma análoga ao início da deformidade plástica de um sistema de metais.

A análise estatística para os testes biomecânicos foi realizada por meio de análise de variância (ANOVA, em inglês) com teste de múltiplas comparações de Tukey da diferença honestamente significativa (honestly significant difference, (HSD), considerando-se valores de $p \leq 0,05$.

\section{Resultados}

Durante a fase de acomodação do enxerto, descrita como toeregion, até $35 \mathrm{~N}$ houve uma rigidez menor no grupo poste $(23,72 \pm 2,16 \mathrm{~N} / \mathrm{mm})$, seguido pelo grupo interferência $(33,52 \pm 2,23 \mathrm{~N} / \mathrm{mm})$ e pelo grupo híbrido $(37,13 \pm 2,37$ $\mathrm{N} / \mathrm{mm}$ ). Não houve diferença estatisticamente significativa entre os grupos interferência e híbrido. Já em relação ao sistema tipo poste (grupo poste) e tipo pressão (grupos interferência e híbrido), houve diferenças estatisticamente significativas ( $p<0,05)$ (-Figura 4 ).

A rigidez do sistema até a falha foi maior no grupo híbrido $(59,10 \pm 3,45 \mathrm{~N} / \mathrm{mm})$. A fixação do grupo poste foi a que atingiu a menor rigidez $(35,75 \pm 3,15 \mathrm{~N} / \mathrm{mm})$, menor do que a fixação com o parafuso de interferência $(40,26 \pm 3,24 \mathrm{~N} / \mathrm{mm})$. Não houve diferença estatisticamente significativa entre os grupos interferência e poste no cálculo de rigidez pela linearidade do gráfico (método tangente). Ambas, no entanto, foram menores do que a do sistema híbrido ( $p<0,05$ ) (-Figura 5).

Verificou-se uma carga para falha no grupo interferência de $270,34 \pm 31,45 \mathrm{~N}$; para o grupo poste, ela foi de $463,72 \pm 30,57 \mathrm{~N}$, e, para o grupo híbrido, de $581,34 \pm 33,49 \mathrm{~N}$. A análise estatística revelou maior resistência do grupo híbrido $(p<0,05)$, seguido pelo grupo poste, e, por fim, o grupo interferência (-Figura 6 ). Todas as falhas

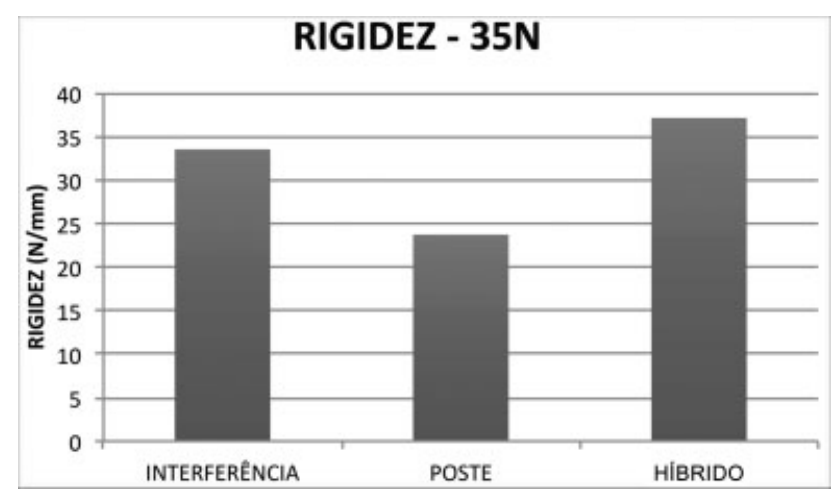

Fig. 4 Resultados de rigidez até 35 N (N/mm).

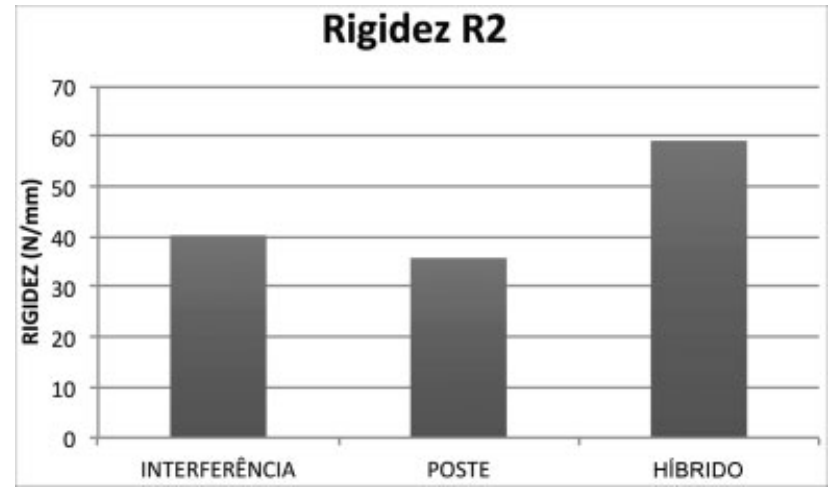

Fig. 5 Resultados da rigidez final do sistema (N/mm).

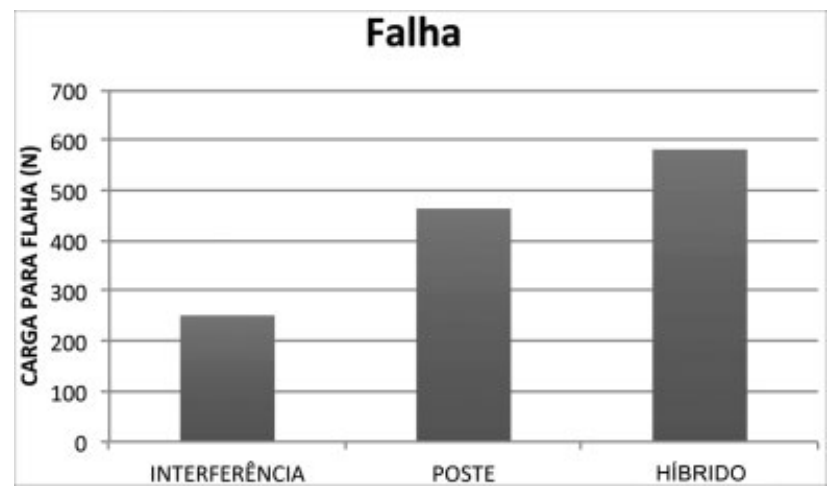

Fig. 6 Resultados de falha do sistema (yield load) (N).

ocorreram por escorregamento e/ou ruptura da sutura, na fixação tipo poste.

A tensão para falha do grupo híbrido $(18,89 \pm 1,66 \mathrm{KPa})$ não apresentou diferença estatisticamente significativa com relação ao grupo com fixação tipo poste $(15,76 \pm 1,61 \mathrm{KPa})$, mas ambos se mostraram estatisticamente diferentes quando comparados com a fixação do grupo interferência $(8,31 \pm 0,87 \mathrm{KPa})(p<0,05)$ (-Figura 7).

\section{Discussão}

O presente estudo foi executado utilizando o tendão digital profundo bovino implantado em tíbia de porco, estruturas com similaridades biomecânicas com a estrutura humana. ${ }^{21,22}$

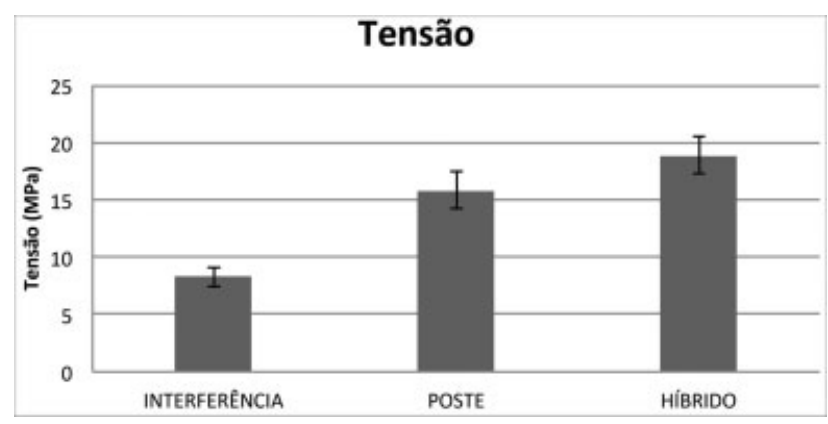

Fig. 7 Resultados de tensão para falha do sistema (Mpa). 
A fixação dos tendões foi realizada com parafuso de interferência (grupo interferência); poste com parafuso, arruela e amarrilho (grupo poste); e fixação com combinação das técnicas(grupo híbrido).

Com o objetivo de analisar se a fixação híbrida apresenta vantagens biomecânicas sobre as demais, cada corpo de prova foi submetido a ensaio de tração de ciclo único (pullout test), com orientação da carga paralela ao túnel ósseo tibial, e determinação da rigidez do sistema e carga para falha (yield load).

Durante os ensaios de tração, verificou-se que, na fase de acomodação do enxerto, a rigidez no grupo poste $(23,72 \pm 2,16 \mathrm{~N} / \mathrm{mm})$ foi menor em relação às demais (fixação tipo pressão: $33,52 \pm 2,23 \mathrm{~N} / \mathrm{mm}$; fixação híbrida: $37,13 \pm 2,37 \mathrm{~N} / \mathrm{mm})$. Grande parte dos estudos descarta esta fase do ensaio para avaliação biomecânica da fixação; ${ }^{10}$ porém, estas cargas fazem parte do processo de reabilitação após a cirurgia de reconstrução do LCA. ${ }^{19}$ Havendo uma rigidez baixa do sistema de fixação tipo poste, é possível um prejuízo da sua cicatrização em relação aos métodos que usam fixação tipo pressão. Uma hipótese para esse achado é a de que a fixação tipo pressão permite menos acomodação inicial do que o tipo poste.

O método de fixação híbrida mostrou rigidez superior aos demais grupos $(59,10 \pm 3,45 \mathrm{~N} / \mathrm{mm})$, com significância estatística, sendo a rigidez o parâmetro biomecânico que melhor se relaciona com o grau de frouxidão observado no exame clínico pós-operatório. ${ }^{7}$ Estes achados sugerem que, no pós-operatório imediato, o método híbrido permite maior rigidez do sistema quando comparado ao método de fixação com parafuso de interferência ou poste. ${ }^{23}$

A carga para falha (yield load) na fixação híbrida $(581,34 \pm 33,49 \mathrm{~N})$ mostrou ser superior em relação ao grupo interferência $(270,34 \pm 31,45 \mathrm{~N})$, com diferença estatisticamente significativa, o que está de acordo com outros estudos. ${ }^{14,22}$ Uma hipótese para esse aumento de resistência é a de que a fixação cortical previne o escorregamento do tendão com maior eficácia do que a fixação por pressão.

Considerando a necessidade de uma resistência da fixação de 445 N no pós-operatório da reconstrução do LCA, ${ }^{24}$ apenas a fixação híbrida mostrou-se segura dentro destes parâmetros. Os resultados mostram que a fixação apenas com parafuso de interferência pode não ser suficientemente segura, não só pelos valores de baixa resistência, como pela variação dos resultados.

A tensão para a falha do grupo híbrido $(18,89 \pm 1,66 \mathrm{KPa})$ não mostrou diferença estatisticamente significativa com relação à do grupo com fixação tipo poste $(15,76 \pm 1,61$ $\mathrm{KPa})$, mas ambos apresentaram diferença estatística significativa com relação à do grupo com fixação tipo interferência $(8,31 \pm 0,87 \mathrm{KPa})$, o que mostra concordância com os achados da resistência pela carga para falha (yield load), não havendo influência objetiva do diâmetro dos tendões utilizados como enxertos em cada grupo. Uma hipótese para isso é a de que os tendões tinham pouca variação de espessura, uma vez que todos tinham a mesma origem, e os que não eram passíveis de serem usados em túnel de nove milímetros não podiam ser usados no experimento.
Estes achados revelam que, na fixação híbrida, ocorre a soma de benefícios dos métodos de fixação, mantendo uma rigidez adequada nas primeira e última fases do ensaio, com carga e tensão para falha superiores às dos demais métodos.

O presente estudo tem limitações, como o fato de que uso de modelos animais, mesmo que aceitável, não substitutui de forma plena o osso humano jovem; portanto, os resultados desta pesquisa não podem ser extrapolados em valores absolutos para a cirurgia em humanos. ${ }^{22}$ Além disso, o uso do ensaio de ciclo único (pull-out test), apesar de ter valor científico, subestima o alongamento e a consequente falha do sistema de fixação encontrados em ensaios cíclicos. $^{25}$

Estudos futuros com testes de carga cíclica são recomendados para analisar a taxa de deformação e escorregamento dos sistemas.

\section{Conclusão}

A fixação tibial híbrida, para reconstrução do LCA, em modelo animal, tem vantagens biomecânicas com relação à fixação simples com parafuso de interferência ou poste no momento do pós-operatório imediato.

Conflitos de Interesse

Os autores declaram não haver conflitos de interesse.

\section{Referências}

$1 \mathrm{Fu} \mathrm{FH}$, Bennett $\mathrm{CH}$, Lattermann C, Ma CB. Current trends in anterior cruciate ligament reconstruction. Part 1: Biology and biomechanics of reconstruction. Am J Sports Med 1999;27(06): 821-830

2 Sanders TL, Maradit Kremers H, Bryan AJ, et al. Incidence of Anterior Cruciate Ligament Tears and Reconstruction: A 21-Year PopulationBased Study. Am J Sports Med 2016;44(06):1502-1507

3 Majewski M, Susanne H, Klaus S. Epidemiology of athletic knee injuries: A 10-year study. Knee 2006;13(03):184-188

4 Astur DC, Xerez M, Rozas J, Debieux PV, Franciozi CE, Cohen M. Lesões do ligamento cruzado anterior e do menisco no esporte: incidência, tempo de prática até a lesão e limitações causadas pelo trauma. Rev Bras Ortop 2016;51(06):652-656

5 Domnick C, Raschke MJ, Herbort M. Biomechanics of the anterior cruciate ligament: Physiology, rupture and reconstruction techniques. World J Orthop 2016;7(02):82-93

6 De Wall M, Scholes CJ, Patel S, Coolican MRJ, Parker DA. Tibial fixation in anterior cruciate ligament reconstruction: a prospective randomized study comparing metal interference screw and staples with a centrally placed polyethylene screw and sheath. Am J Sports Med 2011;39(09):1858-1864

7 Steiner ME, Hecker AT, Brown CH Jr, Hayes WC. Anterior cruciate ligament graft fixation. Comparison of hamstring and patellar tendon grafts. Am J Sports Med 1994;22(02):240-246, discussion 246-247

8 Scheffler SU, Südkamp NP, Göckenjan A, Hoffmann RFG, Weiler A. Biomechanical comparison of hamstring and patellar tendon graft anterior cruciate ligament reconstruction techniques: The impact of fixation level and fixation method under cyclic loading. Arthroscopy 2002;18(03):304-315

9 Brand J Jr, Weiler A, Caborn DN, Brown CH Jr, Johnson DL. Graft fixation in cruciate ligament reconstruction. Am J Sports Med 2000;28(05):761-774 
10 Prado M, Martín-Castilla B, Espejo-Reina A, Serrano-Fernández JM, Pérez-Blanca A, Ezquerro F. Close-looped graft suturing improves mechanical properties of interference screw fixation in ACL reconstruction. Knee Surg Sports Traumatol Arthrosc 2013;21(02):476-484

11 Rodeo SA, Arnoczky SP, Torzilli PA, Hidaka C, Warren RF. Tendonhealing in a bone tunnel. A biomechanical and histological study in the dog. J Bone Joint Surg Am 1993;75(12):1795-1803

12 Eguchi A, Ochi M, Adachi N, Deie M, Nakamae A, Usman MA. Mechanical properties of suspensory fixation devices for anterior cruciate ligament reconstruction: comparison of the fixed-length loop device versus the adjustable-length loop device. Knee 2014; 21(03):743-748

13 Kousa P, Järvinen TLN, Vihavainen M, Kannus P, Järvinen M. The fixation strength of six hamstring tendon graft fixation devices in anterior cruciate ligament reconstruction. Part II: tibial site. Am J Sports Med 2003;31(02):182-188

14 Tetsumura S, Fujita A, Nakajima M, Abe M. Biomechanical comparison of different fixation methods on the tibial side in anterior cruciate ligament reconstruction: a biomechanical study in porcine tibial bone. J Orthop Sci 2006;11(03):278-282

15 Lee JJ, Otarodifard K, Jun BJ, McGarry MH, Hatch GF III, Lee TQ. Is supplementary fixation necessary in anterior cruciate ligament reconstructions? Am J Sports Med 2011;39(02):360-365

16 Stieven Filho E, Malafaia O, Ribas-Filho JM, et al. [Biomechanic analysis of the sewed tendons for the reconstruction of the anterior cruciate ligament]. Rev Col Bras Cir 2010;37(01):52-57

17 Woo SL, Hollis JM, Adams DJ, Lyon RM, Takai S. Tensile properties of the human femur-anterior cruciate ligament-tibia complex. The effects of specimen age and orientation. Am J Sports Med 1991;19(03):217-225

18 Scannell BP, Loeffler BJ, Hoenig M, et al. Biomechanical comparison of hamstring tendon fixation devices for anterior cruciate ligament reconstruction: Part 2. Four tibial devices. Am J Orthop 2015;44(02):82-85

19 Trump M, Palathinkal DM, Beaupre L, Otto D, Leung P, Amirfazli A. In vitro biomechanical testing of anterior cruciate ligament reconstruction: traditional versus physiologically relevant load analysis. Knee 2011;18(03):193-201

20 Aga C, Rasmussen MT, Smith SD, et al. Biomechanical comparison of interference screws and combination screw and sheath devices for soft tissue anterior cruciate ligament reconstruction on the tibial side. Am J Sports Med 2013;41(04):841-848

21 Donahue TL, Gregersen C, Hull ML, Howell SM. Comparison of viscoelastic, structural, and material properties of double-looped anterior cruciate ligament grafts made from bovine digital extensor and human hamstring tendons. J Biomech Eng 2001;123(02): 162-169

22 Nagarkatti DG, McKeon BP, Donahue BS, Fulkerson JP. Mechanical evaluation of a soft tissue interference screw in free tendon anterior cruciate ligament graft fixation. Am J Sports Med 2001;29(01):67-71

23 Fabbriciani C, Mulas PD, Ziranu F, Deriu L, Zarelli D, Milano G. Mechanical analysis of fixation methods for anterior cruciate ligament reconstruction with hamstring tendon graft. An experimental study in sheep knees. Knee 2005;12(02):135-138

24 Zantop T, Weimann A, Rümmler M, Hassenpflug J, Petersen W. Initial fixation strength of two bioabsorbable pins for the fixation of hamstring grafts compared to interference screw fixation: single cycle and cyclic loading. Am J Sports Med 2004;32(03): 641-649

25 Giurea M, Zorilla P, Amis AA, Aichroth P. Comparative pull-out and cyclic-loading strength tests of anchorage of hamstring tendon grafts in anterior cruciate ligament reconstruction. Am J Sports Med 1999;27(05):621-625 